\title{
The Design of an Integrated Control System in Heavy Vehicles Based on an LPV Method
}

\author{
Péter Gáspár, Zoltán Szabó and József Bokor
}

\begin{abstract}
In this paper an integrated control structure with individual active control mechanisms, i.e. active anti-roll bars, active suspensions, and an active brake mechanism, is proposed. Its purpose is to improve rollover prevention, passenger comfort, road holding and guarantee the suspension working space. In the control design the performance specifications both for rollover and suspension problems, and the model uncertainties are taken into consideration. In the weighting strategy of control design fault information is also taken into consideration. The design of the control system is based on an $H_{\infty}$ Linear Parameter Varying (LPV) method. To enhance the performance of the controlled system, the control mechanism is extended with a prediction procedure, in which an observerbased prediction algorithm is proposed.
\end{abstract}

\section{INTRODUCTION}

Several individual active control mechanisms are applied in road vehicles to solve different control tasks. In this paper an integrated control mechanism is proposed, which creates a balance between different components in order to enhance the performance behavior and safety of the vehicle. The control mechanism includes an active suspension, active anti-roll bars, and an active brake. The role of the active suspension system is to improve passenger comfort, road holding and guarantee the suspension working space. The role of the active anti-roll bars is to generate a stabilizing moment in order to balance the overturning moments generated during maneuvers. By using the brake system rollover prevention can be guaranteed, since it reduces directly the lateral tire forces and decelerates the vehicle.

The goal is to design an integrated controller that uses the active suspension and the active anti-roll bars all the time in order to enhance passenger comfort, improve road holding and guarantee the suspension working space. It activates the controlled braking system only when the vehicle comes close to rolling over. The brake is activated only in emergencies. In this paper the detection of an imminent rollover is based on the monitoring of the lateral load transfers for both axles. If the lateral load transfer takes on a critical value, the brake is activated.

This control structure also results in a fault-tolerant system. In order to enhance rollover prevention, the active

This work was supported by the Hungarian National Office for Research and Technology through the project "Advanced Vehicles and Vehicle Control Knowledge Center" (OMFB-01418/2004) and the Hungarian National Science Foundation (OTKA) under the grant $T-048482$ which are gratefully acknowledged. The authors were supported by the János Bolyai Research Scholarship of the Hungarian Academy of Sciences.

P. Gáspár, Z. Szabó and J. Bokor are with the Systems and Control Laboratory, Computer and Automation Research Institute, Hungarian Academy of Sciences, Hungary, Phone: 361-2796171, Fax: 361-4667503, E-mail: gaspar@sztaki.hu suspension assumes the role of the active anti-roll bars if a hydraulic actuator fault occurs in the active bars. In this paper it is assumed that the actuator failure is detected by a Fault Detection and Isolation (FDI) filter, which sends fault information to the combined control.

Several schemes concerned with the possible active intervention into vehicle dynamics have been proposed. These approaches employ active anti-roll bars, active steering, or active braking, see [1], [12], [14], [16], [18], [19]. A combined control structure, in which active anti-roll bars are combined with an active brake control in order to reduce the risk of rollovers, has also been developed, see [8]. There are many papers concerned with different approaches that develop active suspension systems, see [2], [4], [5], [7], [9], [13]. An FDI filter design for vehicle systems has been proposed by several papers, see e.g. [10], [11].

In this paper the yaw, roll and vertical dynamics of the full-car model is constructed in a Linear Parameter Varying (LPV) structure. The control inputs are generated by active anti-roll bars, active suspensions and an active brake. In this paper, the imminent rollover is detected by monitoring the lateral load transfers for both axles. In the control design the performance specifications both for rollover and suspension problems, the model uncertainties and the fault information of the FDI filter are also taken into consideration. In the closed-loop interconnection system three parameters are selected as scheduling variables: the forward velocity, the estimated lateral load transfer and a fault parameter from an FDI filter.

When a rollover is imminent, the switching of the brake may cause a dangerous skid of the vehicle. In this paper the LPV control of the active brake system is extended with a new prediction procedure, in which the critical values are predicted in advance using a short time interval. That is, the values of the lateral load transfer are predicted in order to determine whether these values may exceed the critical value corresponding to the rollover. If in this short time interval the predicted values exceed the critical lateral load transfer, the brake is activated.

The structure of the paper is as follows. In Section $I I$ the LPV model of the yaw-roll and suspension dynamics are presented. In Section $I I I$ an observer based prediction algorithm is proposed for the detection of an imminent rollover. In Section $I V$ the weighting strategy in the fault-tolerant control structure is defined. In Section $V$ the the operation of the fault-tolerant control is demonstrated through simulation examples. Finally, Section VI contains some concluding remarks. 


\section{THE MODELLING OF FULL-CAR VEHICLE DYNAMICS}

An integrated control using three control mechanisms to handle suspension and rollover problems is proposed. If all the control mechanisms are to be designed simultaneously, a high-complexity model to be controlled can be formalized, which includes a complex model with pitch, roll and vertical motions and in the control design a large number of performance demands should be handled at the same time. However, the handling of this control problem is numerically difficult. Consequently the complex control design problem is divided into two subtasks: a task for the suspension design and a task for the rollover problem. Two control systems are designed to tackle these problems.

Figure 1 illustrates the combined yaw-roll dynamics of the vehicle, which is modelled by a three-body system, in which $m_{s}$ is the sprung mass, $m_{u, f}$ is the unsprung mass at the front including the front wheels and axle, $m_{u, r}$ is the unsprung mass at the rear. The signals are the lateral acceleration $a_{y}$, the side slip angle of the sprung mass $\beta$, the heading angle $\psi$, the yaw rate $\dot{\psi}$, the roll angle $\phi$, the roll rate $\dot{\phi}$, the roll angle of the unsprung mass at the front axle $\phi_{t, f}$ and at the rear axle $\phi_{t, r} . \delta_{f}$ is the front wheel steering angle. $v$ is the forward velocity. This structure includes three control mechanism, which generate control inputs. They are the roll moments between the sprung and unsprung mass generated by active anti-roll bars $u_{a f}$ and $u_{a r}$, the difference in brake forces between the left and righthand side of the vehicle $\Delta F_{b}$, and forces between the sprung and unsprung masses generated by active suspensions $f_{1}$ and $f_{2}: u_{r}=\left[\begin{array}{lllll}u_{a f} & u_{a r} & f_{1} & f_{2} & \Delta F_{b}\end{array}\right]^{T}$. The relationship between the actual suspension forces and the fictitious forces is $f_{1}=f_{f l}-f_{f r}$ and $f_{2}=f_{r l}-f_{r r}$.

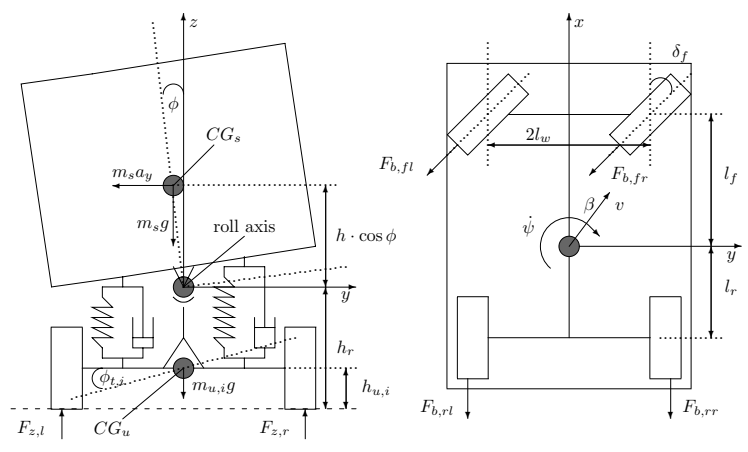

Fig. 1. The yaw-roll model for control design

In the modelling of vertical dynamics a simplified half-car model, which is shown in Figure 2, is substituted for the fullcar model, since the roll motion of the vehicle is handled in the modelling of the rollover system. The sprung mass $m_{s}$ is assumed to be a rigid body and has freedoms of motion in the vertical and pitch directions. $x_{1}$ denotes the vertical displacement at the center of gravity and $\theta$ is the pitch angle of the sprung mass. The front and rear displacements of the unsprung masses are denoted by $x_{2 f}, x_{2 r}$, respectively. The disturbances in the suspension system are caused by road irregularities $w_{f}, w_{r}$. Here two fictitious input forces, $f_{3}$ and $f_{4}$, are generated by the actuators: $u_{s}=\left[\begin{array}{ll}f_{3} & f_{4}\end{array}\right]^{T}$. The relationship between the actual suspension forces and the fictitious forces are the following: $f_{3}=f_{f l}+f_{f r}, f_{4}=$ $f_{r l}+f_{r r}$. It is important to note that although the control design for the suspension system is based on the half-car model and the performance signals are the heave and the pitch accelerations, the control system designed is operated on the whole full-car model.

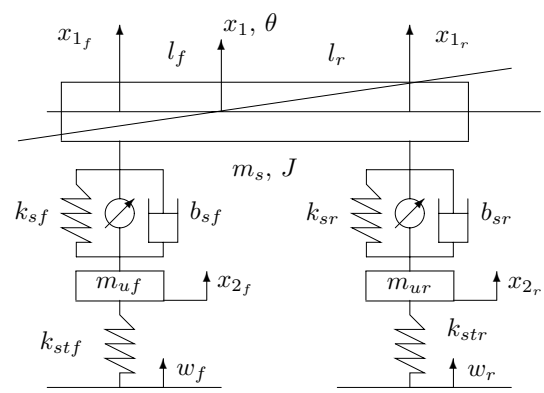

Fig. 2. The half-car model for suspension design

The motion differential equations can be formalized both for the rollover and the suspension dynamics, and by selecting state variables the state space representation can be formalized, see [8]:

$$
\begin{aligned}
& \dot{x}_{r}=A_{r}(v) x_{r}+B_{1 r}(v) \delta_{f}+B_{2 r}(v) u_{r} \\
& \dot{x}_{s}=A_{s} x_{s}+B_{1 s} w+B_{2 s} u_{s}
\end{aligned}
$$

with $x_{r}=\left[\begin{array}{llllll}\beta & \dot{\psi} & \phi & \dot{\phi} & \phi_{t, f} & \phi_{t, r}\end{array}\right]^{T}$ and $x_{s}=\left[\begin{array}{llllllll}x_{1} & \theta & x_{2 f} & x_{2 r} & \dot{x_{1}} & \dot{\theta} & x_{2 f} & x_{2 r}\end{array}\right]^{T}$.In the equation (1) the system matrices depend on the forward velocity of the vehicle nonlinearly. If $v$ is chosen as a scheduling parameter, the differential equations of the yawroll motion are linear in the state variables: $\rho=v$. The disturbance signals are the front wheel steering angle $\delta_{f}$, and the road disturbances $w_{f}, w_{r}$.

Note that the fictitious control signals $f_{1}, f_{2}$ are in $u_{r}$ and $f_{3}, f_{4}$ are $u_{s}$. These fictitious signals are generated by the actual suspension forces. Based on the relationship between the actual suspension forces and the fictitious suspension forces, the actual forces can be calculated in a simple matrix manipulation:

$$
\begin{aligned}
& f_{f l}=\frac{f_{1}+f_{3}}{2}, f_{f r}=\frac{f_{3}-f_{1}}{2}, \\
& f_{r l}=\frac{f_{2}+f_{4}}{2}, f_{r r}=\frac{f_{4}-f_{2}}{2} .
\end{aligned}
$$

\section{OBSERVER BASED PREDICTION OF THE LATERAL LOAD TRANSFERS}

Roll stability is achieved by limiting the lateral load transfers for both axles, $\Delta F_{z, l}$ and $\Delta F_{z, r}$, to below the levels for wheel lift-off. The lateral load transfer is calculated: $\Delta F_{z, i}=\frac{k_{t, i} \phi_{t, i}}{l_{w}}$, where $k_{t, i}$ is the stiffness of the tires at the front and rear axles, $\phi_{t, i}$ is the roll angle of the unsprung 
mass and $l_{w}$ is the vehicle's width, and $i$ denotes the front and rear of the vehicle.

The tire contact force is guaranteed if $\frac{m g}{2} \pm \Delta F_{z}>0$ for both sides of the vehicle. This requirement leads to the definition of the normalized load transfer, which is the ratio of the lateral load transfers at the front and rear axles:

$$
R_{i}=\frac{\Delta F_{z, i}}{m_{i} g} .
$$

where $m_{i}$ is the mass of the vehicle in the front and the rear. The normalized load transfer $R_{i}$ value corresponds to the largest possible load transfer. If the $R_{i}$ takes on the value \pm 1 then the inner wheels in the bend lift off. The limit cornering condition occurs when the load on the inside wheels has dropped to zero and all the load has been transferred onto the outside wheels.

In this paper the detection of an imminent rollover is based on the monitoring of the lateral load transfers for both axles. In the control design the predicted value of the normalized load transfer is used. It is generated in two steps:

Step 1 : The design of an observer to generate $\tilde{R}$ based on the measured signals, $a_{y}, \dot{\psi}$ and $\dot{\phi}$, where $a_{y}$ is the lateral acceleration.

Step 2: Prediction in a short period $\hat{R}$ based on the observed $\tilde{R}$.

It can be shown that for the LPV model of the yaw-roll dynamics the state matrices depend affinely on the parameter vector. This class of systems is described as:

$$
\begin{aligned}
& \dot{x}(t)=A(\rho) x(t)+B_{2}(\rho) u(t) \\
& y(t)=C x(t),
\end{aligned}
$$

where $A(\rho)=A_{0}+\rho_{1} A_{1}+\cdots+\rho_{N} A_{N}, B_{2}(\rho)=B_{0}+$ $\rho_{1} B_{1}+\cdots+\rho_{N} B_{N}$ and $\rho_{i}$ are time varying parameters. It is assumed that each parameter $\rho_{i}$ and its derivatives $\dot{\rho}_{i}$ range between known extremal values $\rho_{i}(t) \in\left[-\bar{\rho}_{i}, \bar{\rho}_{i}\right]$ and $\dot{\rho}_{i}(t) \in\left[-\overline{\dot{\rho}}_{i}, \bar{\rho}_{i}\right]$, respectively. Let us denote this parameter set by $\mathcal{P}$. For the model of the yaw-roll dynamics $N=2$ and the parameters are $\rho_{1}=v$ and $\rho_{2}=\frac{1}{v}$.

In order to make a prediction for the lateral load transfers the roll angles of the unsprung masses $\phi_{t, i}$ must be estimated. A Luenberger type observer for the system is proposed. The form of the observer is the following:

$$
\dot{\eta}=(A(\rho)+K(\rho) C) \eta+B(\rho) u-K(\rho) y .
$$

Concerning the question of stability, let us recall that an LPV system is said to be quadratically stable if there exist a matrix $P=P^{T}>0$ such that

$$
A(\rho)^{T} P+P A(\rho)<0
$$

for all the parameters $\rho \in \mathcal{P}$. A necessary and sufficient condition for a system to be quadratically stable is that the condition in equation (8) should hold for all the corner points of the parameter space, i.e., one can obtain a finite system of LMI's that has to be fulfilled for $A(\rho)$ with a suitable positive definite matrix $P$, see [6], [21].

In order to obtain a quadratically stable observer the LMI $(A(\rho)+K(\rho) C)^{T} P+P(A(\rho)+K(\rho) C)<0$ must hold for suitable $G(\rho)$ and $P=P^{T}>0$. By introducing the auxiliary variable $G(\rho)=P K(\rho)$, one has to solve the following set of LMIs on the corner points of the parameter space:

$$
A(\rho)^{T} P+P A(\rho)+C^{T} K(\rho)^{T}+K(\rho) C<0 .
$$

By solving these LMIs one can obtain a suitable observer gain. The obtained observer gives us an estimation for the state vector, hence, an estimation for the values of the roll angles of the unsprung masses $\phi_{t, i}$. With these values the normalized load transfers $\tilde{R}_{i}$ are calculated. These values will be considered as input data for the prediction step.

Since the inputs of the model are not known in advance it is considered to be more reliable to use a simple linear model, e.g., an AR model structure, to model only the evolution of the normalized load transfer based on measurements of a given length. Therefore the prediction algorithm fits an AR model structure of order $n$ to the sampled values of length $\ell_{n}$ and then using this model it predicts the values of $\hat{R}_{i}$ for a horizon of $\ell_{h}$ samples. For implementation a recursive least squares estimation method is used.

The problem in this setting is to estimate the parameter $\theta_{0}$ in the equation

$$
y(t)=\psi^{T}(t) \theta_{0}+e(t),
$$

in order to minimize $\sum_{t=1}^{T}=\lambda^{T-t}\|e(t)\|^{2}$, where $\psi^{T}(t)$ is the regression vector with known values and $\lambda$ is a forgetting factor that sets the discount of the old measurements exponentially. The recursive estimation algorithm can be summarized as

$$
\begin{aligned}
\hat{\theta}(t) & =\hat{\theta}(t-1)+K(t)[y(t)-\hat{y}(t)], \\
K(t) & =Q(t) \Psi(t), \\
Q(t) & =\frac{P(t-1)}{\lambda+\Psi^{T}(t) P(t-1) \Psi(t)}, \\
P(t) & =\frac{1}{\lambda}\left[P(t-1)-\frac{P(t-1) \Psi(t) \Psi^{T}(t) P(t-1)}{\lambda+\Psi^{T}(t) P(t-1) \Psi(t)}\right],
\end{aligned}
$$

where $\hat{y}(t)=\Psi^{T}(t) \hat{\theta}(t-1)$, for details see [15].

Based on the recursive algorithm $\hat{R}_{r}$ is estimated. The prediction is based on values taken within a short time interval. The model order is set at $n=2, \ell_{n}$ consists of 50 sampling points and $\ell_{h}$ is chosen to be 5 sampling points. The forgetting factor $\lambda$ in the recursive AR algorithm is set at 0,97 . The value of $\ell_{h}$ provides a prediction interval between $0.5 \mathrm{~m}$ and $2 \mathrm{~m}$ depending on the forward velocity. In the control design step the predicted values of the normalized load transfer $\hat{R}_{r}$ are applied instead of the value $R_{r}$ at the time $t_{i}$. Here $\hat{R}_{r}$ is the predicted value at the time $t_{i+\ell_{h}}$.

\section{THE DESIGN OF A WEIGHTING STRATEGY IN THE INTEGRATED CONTROL}

In the control design both the rollover and the suspension problems are taken into consideration. The performance demands are usually in contradiction and a balance between these specifications must be achieved. In this combined structure a a new weighting strategy is proposed in order to meet 
several performance demands, such as enhancing passenger comfort, rollover stability and road holding, guaranteing suspension working space and reducing energy consumption.

In the rollover problem the performance outputs for control design are the lateral acceleration, the lateral load transfers at the front and the rear, and the control inputs: $z_{r}=\left[\begin{array}{llll}a_{y} & \Delta F_{z, f} & \Delta F_{z, r} & u_{r}\end{array}\right]^{T}$.In the suspension problem the performance outputs for control design are passenger comfort (i.e. heave acceleration), the suspension deflections, the tire deflections and the control inputs: $z_{s}=$ $\left[\begin{array}{llllll}a_{z} & z_{s f} & z_{s r} & z_{t f} & z_{t r} & u_{s}\end{array}\right]^{T}$. The measured outputs are the lateral acceleration of the sprung mass, the derivative of the roll angle and the suspension deflections at the suspension components: $y_{r}=\left[\begin{array}{ll}a_{y} & \dot{\phi}\end{array}\right]^{T}, y_{s}=\left[\begin{array}{ll}z_{s f} & z_{s r}\end{array}\right]^{T}$. Although the performance signals are formalized to the full-car model, the design is performed in two subsystems instead of the fullcar model. One of them is the yaw-roll model for rollover prevention, which is controlled by $u_{r}$ and $\Delta F_{b}$. The second subsystem is the half-car model for suspension design, which is controlled by $u_{s}$.

The closed-loop interconnection structures both for rollover prevention and suspension design can be seen in Figure 3 and 4. Two controllers are designed independently of each other. The design of $K_{r}$ is based on the yaw and roll model and the performance specifications for the rollover prevention system. $K_{r}$ creates the roll moments of the active anti-roll bars $u_{a f}, u_{a r}$, the fictitious control forces of the active suspensions $u_{s 1}, u_{s 2}$, and the difference between brake forces $\Delta F_{b}$. The design of $K_{s}$ is based on the vertical dynamics of the vehicle and the performance specifications for the suspension system. $K_{s}$ creates the fictitious control forces of the active suspensions $u_{s 3}$ and $u_{s 4}$.

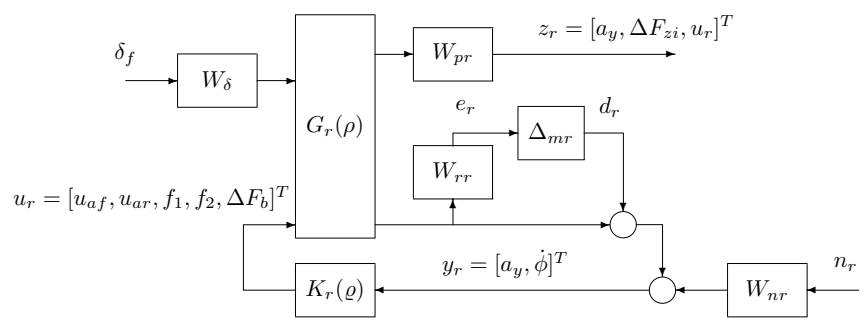

Fig. 3. The closed-loop interconnection structure for the design of a rollover prevention system

The weighting functions for performances $W_{p r}$ and $W_{p s}$ are considered as penalty functions. The uncertainties of the nominal model are represented by a multiplicative structure. The uncertainties of the nominal model are represented by the weighting function $W_{r r}$ and $W_{r s}$. The input scaling weights $W_{\delta}$ and $W_{w}$ normalize the disturbances to the maximum expected command. The weighting functions $W_{n r}$ and $W_{n s}$ take the sensor noises into account in the control design. In this paper three performances, which are significant in the integrated control, are highlighted. These are the lateral acceleration $a_{y}$, the lateral load transfers $\Delta F_{z i}$ and the heave acceleration $a_{z}$. All of these weighting functions are selected

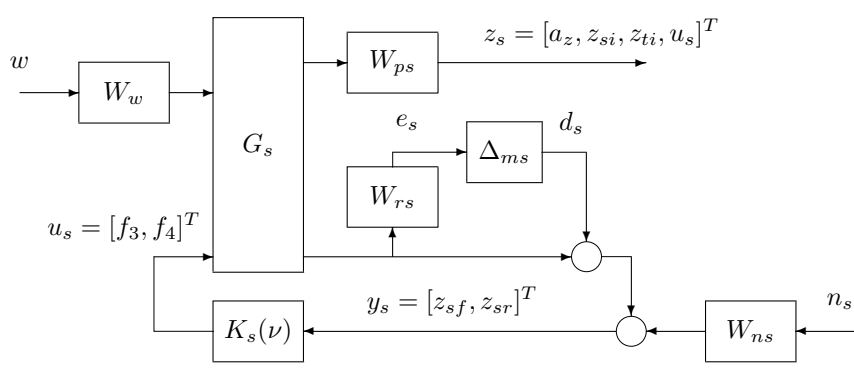

Fig. 4. The closed-loop interconnection structure for the design of a suspension system

in such a way that they depend on the normalized lateral load transfer $R$ :

$$
\begin{aligned}
& W_{p, a y}=\phi_{a y} \frac{\frac{s}{2000}+1}{\frac{s}{12}+1}, \\
& W_{p, F z}=\operatorname{diag}\left(\frac{\phi_{F_{z}}}{7}, \frac{\phi_{F_{z}}}{10}\right), \\
& W_{p, a z}=\phi_{a z}\left(1-\rho_{f}\right) \frac{0.75\left(\frac{s}{750}+1\right)}{\frac{s}{10}+1},
\end{aligned}
$$

where

$$
\begin{aligned}
& \phi_{a y}(R)=\left\{\begin{array}{cll}
0 & \text { if } & |R|<R_{3} \\
\frac{2\left(|R|-R_{3}\right)}{\left(R_{4}-R_{3}\right)} & \text { if } & R_{3} \leq|R| \leq R_{4} \\
2 & \text { if } & |R|>R_{4}
\end{array}\right. \\
& \phi_{F z}(R)=\left\{\begin{array}{ccc}
0.01 & \text { if } & |R|<R_{1} \\
\frac{2\left(|R|-R_{1}\right)}{\left(R_{2}-R_{1}\right)}+0.01 & \text { if } & R_{1} \leq|R| \leq R_{2} \\
2.01 & \text { if } & |R|>R_{2}
\end{array}\right. \\
& \phi_{a z}(R)=\left\{\begin{array}{ccc}
11 & \text { if } & |R|<R_{1} \\
11-\frac{10\left(|R|-R_{1}\right)}{\left(R_{2}-R_{1}\right)} & \text { if } & R_{1} \leq|R| \leq R_{2} \\
1 & \text { if } & |R|>R_{2}
\end{array}\right.
\end{aligned}
$$

In the lower range of $R$ the gains $\phi_{a y}$ and $\phi_{F z}$ must be small, and in the upper range of $R$ the gains must be large. Consequently, the weighting functions must be selected in such a way that they minimize the lateral acceleration and the lateral load transfers in emergency situations. However in normal cruising situations the control does not focus on the lateral effects since the weight is small. Parameters $R_{1}$ and $R_{3}$ define the critical status when the vehicle is in an emergency situation. Parameters $R_{2}$ and $R_{4}$ show how fast the control should focus on minimizing the lateral effects. In the lower range of $R$ the gain $\phi_{a z}$ must be large, and in the upper range of $R$ the gain must be small. Consequently, the weighting function of $\phi_{a z}$ must be selected in such a way that it minimizes the vertical acceleration in normal cruising mode, and focuses on road holding in emergencies.

$\rho_{f}$ is the the normalized fault parameter, which contains the fault information. Normalization means that the value of a possible fault is normalized into the interval $\left[\begin{array}{ll}0 & 1\end{array}\right]$. The value 0 corresponds to the fault-free operation and the value 1 means that the anti-roll bars are not able to work. If $\rho_{f}$ takes the value between 0 and 1 there is a loss in effectiveness. That is, the active anti-roll bars can be used 
even with reduced power to generate stabilizing moment. In case of a fault the weighting for the active suspension must be modified in such a way that it reduces the rollover risk. When a fault occurs in the active anti-roll bars, the active suspension must assume the role of the active anti-roll bars. In emergency and fault case the role of the active suspensions is to prevent the rollover and passenger comfort is no longer a priority.

In order to describe the control objective, the parameter dependent augmented plant $P_{r}(\varrho)$ and $P_{s}(\nu)$ must be built up using the closed-loop interconnection structure. These augmented plants include the parameter dependent vehicle dynamics and the weighting functions.In the LPV model of $P_{r}(\varrho)$ two parameters are selected: the forward velocity $v$ and the normalized lateral load transfer at the rear side $R$, i.e. $\varrho=\left[\begin{array}{ll}v & R\end{array}\right]$. Here $v$ is measured directly, and parameters $R$ is calculated by using the measured roll angle $\phi_{t, r}$. The scheduling parameter in the LPV model $P_{s}(\nu)$ is $\nu=R$. Note, that although the suspension model is a linear time invariant (LTI), the model of the augmented plant is LPV, because of the weighting strategy.

The closed-loop system $M_{r}(\varrho)$ is given by a lower linear fractional transformation (LFT) structure: $M_{r}(\varrho)=$ $\mathcal{F}_{\ell}\left(P_{r}(\varrho), K_{r}(\varrho)\right)$, where $K_{r}(\varrho)$ depends on the scheduling parameter $\varrho$. The goal of the control design is to minimize the induced $\mathcal{L}_{2}$ norm of a LPV system $M_{r}(\varrho)$, with zero initial conditions, which is given by

$$
\left\|M_{r}(\varrho)\right\|_{\infty}=\sup _{\varrho \in \mathcal{F}_{\mathcal{P}}\left\|w_{r}\right\|_{2} \neq 0, w_{r} \in \mathcal{L}_{2}} \frac{\left\|\tilde{z}_{r}\right\|_{2}}{\left\|w_{r}\right\|_{2}} .
$$

where $w_{r}=\left[\begin{array}{lll}d_{r} & \delta_{f} & n_{r}\end{array}\right]^{T}$ and $\tilde{z}_{r}=\left[\begin{array}{ll}e_{r} & z_{r}\end{array}\right]^{T}$. The formalization of the optimization criterion of the suspension design, i.e. the design of controller $K_{s}(\nu)$ is similar to equations (12). Control of LPV systems with induced $L_{2^{-}}$ norm performance is proposed several authors, see [3], [17], [22], [20].

The controller is constructed by using its two components $K_{r}(\varrho)$ and $K_{s}(\nu)$. These components generate control signals independently of each other. $K_{r}(\varrho)$ creates the roll moments of the active anti-roll bars $u_{a f}, u_{a r}$, the fictitious control forces of the active suspensions $u_{s 1}, u_{s 2}$, and the difference between brake forces $\Delta F_{b} . K_{s}(\nu)$ also creates the fictitious control forces of the active suspensions $u_{s 3}$ and $u_{s 4}$. Based on the fictitious control inputs the actual control forces at the front and rear on both sides generated in the suspension points can be calculated in simple matrix manipulations. Applying the normalized load transfer as a scheduling parameter, the combined control guarantees a balance between improving passenger comfort and enhancing rollover prevention. When the vehicle is in normal cruising mode a control force is generated to improve passenger comfort. When the vehicle is in an emergency, i.e. close to rolling over, a stabilizing moment is generated to balance the overturning moment. Based on the fictitious control inputs the control forces arising at the suspension points are calculated in a simple matrix manipulation according to equation (3).

\section{DEMONSTRATION OF THE FAULT-TOLERANT} CONTROL

In this section the integrated control is illustrated in a cornering maneuver. The velocity of the vehicle is $70 \mathrm{kph}$. The cornering maneuver starts at $1 \mathrm{sec}$ and at $4 \mathrm{sec}$ a bump with a $10 \mathrm{~cm}$ high bump disturbs the motion of the vehicle. The steering angle input is generated in such a way that the vehicle comes close to rolling over during the maneuver and its normalized load transfers are over \pm 1 . The steering angle is a ramp signal. Figure 5 shows the steering angle, the forward velocity, the normalized load transfers, the lateral acceleration, the heave acceleration, the control forces in both normal (solid line) and fault (dashed line) situations.

The time responses in normal cruising case is illustrated by solid line. As the lateral acceleration increases in the cornering, the normalized load transfers also increase. However, the normalized load transfers do not exceed the critical value. The time responses of the heave acceleration occur at 4 sec. Since it is a normal cruising situation, the control focuses on both the minimization of the heave acceleration and the roll stability of the vehicle. In this situation, a control moment $(-40 \mathrm{kNm})$ is generated so that the controller can prevent the rollover of the vehicle. The control also provides good passenger comfort as the heave acceleration shows it. When the effect of the bump results in disturbances on the heave acceleration, a large suspension force generated by the suspension actuators is needed $(12 k N)$ to reduce both the magnitude and the duration of the oscillation. However, the control moments generated by the anti-roll bars are not sufficient to prevent rollovers. When one of the normalized load transfer has reached the critical value $R_{1}$ (at $2 \mathrm{sec}$ ), the brake is also activated and an unilateral braking force ( 5 $k N)$. As a result the velocity of the vehicle decreases and the lateral load transfers are below the critical value 1 .

The time responses of the vehicle in the fault case is illustrated by dashed line. $\rho_{f}$ is assumed to be constant value 1 , i.e. the anti-roll bars have failed completely and are not used. In this case the suspension actuators are used for both passenger comfort and rollover prevention. The lateral acceleration is slightly different in fault and fault-free cases. The reason is that where the normalized load transfers do not reach the critical value, only the active anti-roll bars are activated in a fault-free case and the brake system is not used. However the active anti-roll bars are not able to decrease lateral acceleration because they do not have a direct effect on changing acceleration. So the effect of the control reconfiguration from anti-roll bars to suspension actuators does not have an impact on the lateral acceleration. The normalized load transfers do not exceed the value \pm 1 in any of these cases, which means that the lateral force on one of the curve inner side wheels will not become zero.

In the normal case the control moment generated by the anti-roll bars is $40 \mathrm{kNm}$, and in the fault case the control force generated by the suspension actuators is $24 k N$. In the fault case, when the vehicle has reached the critical lateral load transfer $R_{1}$, the controller focuses only on rollover 

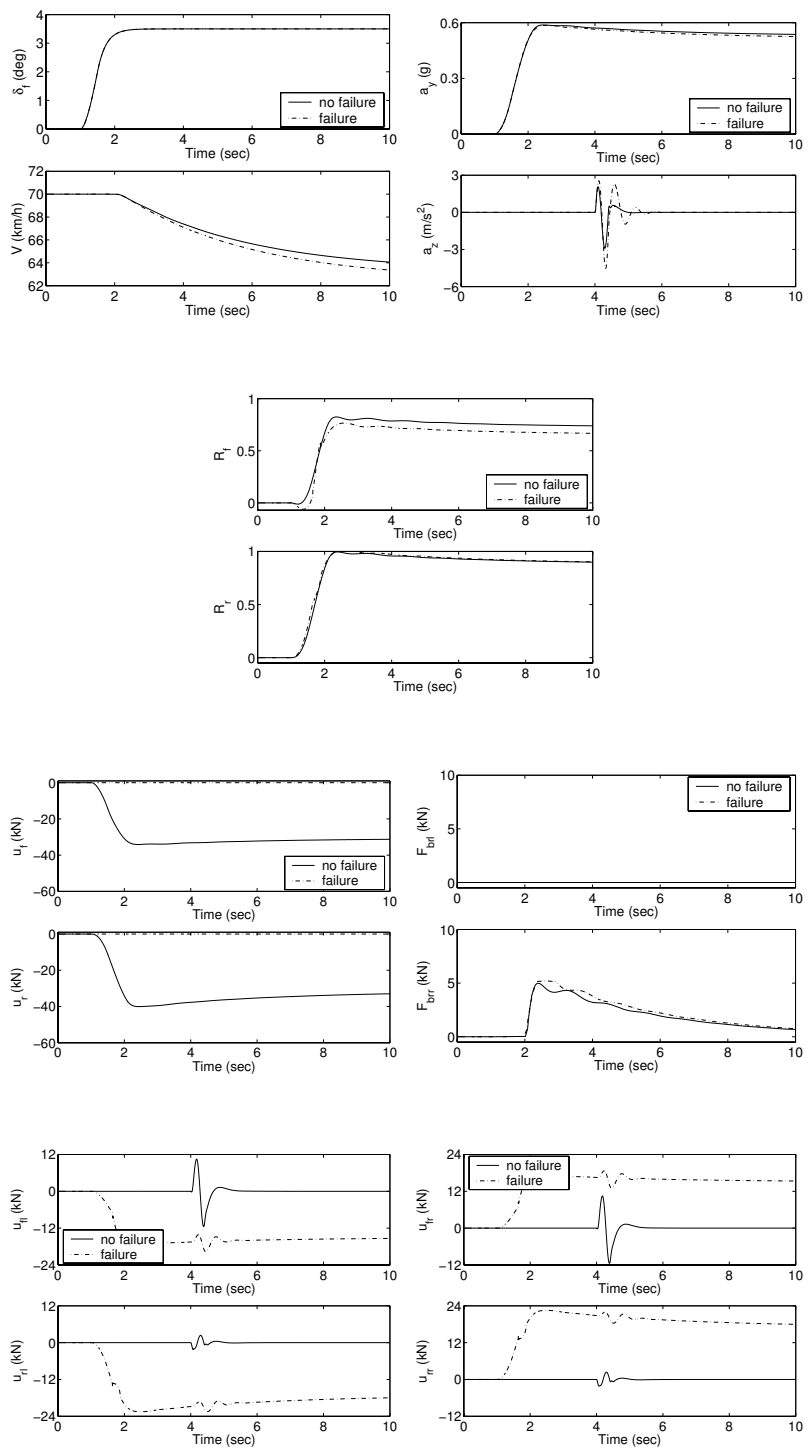

Fig. 5. Time responses to the cornering maneuver in the fault-free and fault situations at $70 \mathrm{kph}$

prevention because the vehicle is close to an emergency. Hence, passenger comfort deteriorates as the heave acceleration shows it. In a normal situation the active anti-roll bars and in the fault case the suspension control is not sufficient to prevent the rollover of the vehicle. Consequently, the normalized load transfers reach the critical value $R_{3}$ during the cornering maneuver so the brake control is activated and the brake system reduces lateral acceleration. The time when the brake control is activated is seen in the brake force figure, which shows that the rear right-hand side wheel is braked to prevent the rollover of the vehicle.

\section{CONCLUSIONS}

In this paper an integrated fault-tolerant controller, which includes active anti-roll bars, active suspensions and an active brake, has been proposed. With this structure both rollover prevention and the passenger comfort can be guaranteed.
Moreover, if a fault occurs in the active anti-roll bars and it is detected by an FDI filter, the active suspension assumes the role of the active anti-roll bars to enhance rollover prevention. A weighting strategy is applied in the closed-loop interconnection structure, in which the normalized lateral load transfers and the residual output of the FDI filter play an important role. This control mechanism guarantees the balance between the active suspension and the active anti-roll bars and balances between rollover prevention and passenger comfort.

\section{REFERENCES}

[1] J. Ackermann and D. Odenthal. Damping of vehicle roll dynamics by gain scheduled active steering. Proc. European Control Conference, 1999.

[2] A. Alleyne and J.K. Hedrick. Nonlinear adaptive control of active suspensions. IEEE Transactions on Control Systems Technology, pages 94-101, 1995.

[3] G. Becker and A. Packard. Robust performance of linear parametrically varying systems using parametrically-dependent linear feedback. System Control Letters, 23:205-215, 1994.

[4] B. de Jager. Multiobjective suspension control problem. Proc. of the 34th Conference on Decision and Control, 4:3652-3657, 1995.

[5] I.J. Fialho and G.J. Balas. Design of nonlinear controllers for active vehicle suspensions using parameter-varying control synthesis. Vehicle System Dynamics, 33:351-370, 2000.

[6] P. Gahinet and P. Apkarian. Explicit controller formulas for lmi-based $\mathcal{H}_{\infty}$ synthesis. Automatica, 32:1007-1014, 1996.

[7] P. Gaspar, I. Szaszi, and J. Bokor. Active suspension design using the mixed $\mu$ synthesis. Vehicle System Dynamics, 40(4):193-228, 2003.

[8] P. Gaspar, I. Szaszi, and J. Bokor. The design of a combined control structure to prevent the rollover of heavy vehicles. European Journal of Control, 10(2):1-15, 2003.

[9] D. Hrovat. Survey of advanced suspension developments and related optimal control applications. Automatica, 33:1781-1817, 1997.

[10] R. Isermann. Real-time fault identification in an active roll control system. Proc. of the 17th IAVSD Symposium, Coppenhagen, 2001

[11] B.P. Jeppesen and D. Cebon. Real-time fault identification in an active roll control system. 17th IAVSD Symposium, Coppenhagen, 2001.

[12] H.J. Kim and Y.P. Park. Investigation of robust roll motion control considering varying speed and actuator dynamics. Mechatronics, 14:35-54, 2004.

[13] J.S. Lin and I. Kanellakopoulos. Nonlinear design of active suspensions. Proc. of the 34th Conference on Decision and Control, 3:35673569, 1995.

[14] R.C. Lin, D. Cebon, and D.J. Cole. Optimal roll control of a singleunit lorry. Proc. IMechE, Journal of Automobile Engineering, pages 44-55, 1996.

[15] L. Ljung. System identification: Theory for the user. Prentice-Hall, Inc., Englewood Cliffs, New Jersey, 1987.

[16] S. Mammar and D. Koenig. Vehicle handling improvement by active steering. Vehicle System Dynamics, 38:211-242, 2002.

[17] A. Packard. Gain scheduling via linear fractional transformations. Systems and Control Letters, 22:79-92, 1994.

[18] L. Palkovics, A. Semsey, and E. Gerum. Roll-over prevention system for commercial vehicles. Vehicle System Dynamics, 32:285-297, 1999.

[19] D.J.M. Sampson and D. Cebon. Active roll control of single unit heavy road vehicles. Vehicle System Dynamics, 40:229-270, 2003.

[20] F. Wu. A generalized LPV system analysis and control synthesis framwework. Int.J. Control, 74:745-759, 2001.

[21] F. Wu, X. H. Yang, A. Packard, and G. Becker. Induced $\mathcal{L}_{2}$-norm control for LPV systems with bounded parameter variation rates. International Journal of Nonlinear and Robust Control, 6:983-998, 1996.

[22] J. Yu and A. Sideris. $\mathcal{H}_{\infty}$ control with parametric Lyapunov functions Systems and Control Letters, 30:57-69, 1997. 